\title{
Multiple Myeloma in the Time of COVID-19
}

\author{
Abdullah S. Al Saleh ${ }^{\mathrm{a}}$ b Taimur Sher ${ }^{\mathrm{c}}$ Morie A. Gertz ${ }^{\mathrm{a}}$ \\ ${ }^{a}$ Division of Hematology, Department of Internal Medicine, Mayo Clinic, Rochester, MN, USA; ${ }^{\text {} K i n g ~ S a u d ~ b i n ~}$ \\ Abdulaziz University for Health Sciences, Riyadh, Saudi Arabia; ${ }^{~}$ Division of Hematology, Department of Internal \\ Medicine, Mayo Clinic, Jacksonville, FL, USA
}

\section{Keywords}

Multiple myeloma · COVID-19 $\cdot$ Recommendations

\begin{abstract}
We provide our recommendations (not evidence based) for managing multiple myeloma patients during the pandemic of COVID-19. We do not recommend therapy for smoldering myeloma patients (standard or high risk). Screening for $\mathrm{CO}$ VID-19 should be done in all patients before therapy. For standard-risk patients, we recommend the following: ixazomib, lenalidomide, and dexamethasone (IRd) (preferred), cyclophosphamide lenalidomide and dexamethasone (CRd), daratumumab lenalidomide and dexamethasone (DRd), lenalidomide, bortezomib, and dexamethasone (RVd), or cyclophosphamide, bortezomib, and dexamethasone (CyBorD). For high-risk patients we recommend carfilzomib, lenalidomide, and dexamethasone (KRd) (preferred) or RVd. Decreasing the dose of dexamethasone to $20 \mathrm{mg}$ and giving bortezomib subcutaneously once a week is recommended. We recommend delaying autologous stem cell transplant (ASCT), unless the patient has high-risk disease that is not responding well, or if the patient has plasma cell leukemia (PCL). Testing for COVID-19 should be done before ASCT. If a
\end{abstract}

patient achieves a very good partial response or better, doses and frequency of drug administration can be modified. After 10-12 cycles, lenalidomide maintenance is recommended for standard-risk patients and bortezomib or ixazomib are recommended for high-risk patients. Daratumumab-based regimens are recommended for relapsed patients. Routine ASCT is not recommended for relapse during the epidemic unless the patient has an aggressive relapse or secondary PCL. Patients on current maintenance should continue their therapy.

c) 2020 S. Karger AG, Basel

\section{Introduction}

The management of patients with cancer during the current pandemic of COVID-19 is challenging. As of March 30, 2020, more than 690,000 confirmed cases and more than 33,000 deaths have been documented worldwide [1]. Cancer patients require special attention, as decisions regarding treatment during the current pandemic require specialized consideration. The quality of care for cancer patients has been affected during this pandemic [2-5] and strategies are required to deliver the best care. karger@karger.com www.karger.com/aha

(C) 2020 S. Karger AG, Base

Karger"
Dr. Morie A. Gertz, MD, MACP

Division of Hematology, Department of Internal Medicine Mayo Clinic, 200 First Street SW

Rochester, MN 55905 (USA)

gertz.morie@mayo.edu 
From a nationwide analysis in China, 18 (1\%) out of 1,590 COVID-19 cases had a history of cancer [6]. Patients with cancer were more likely to be older and had more frequently abnormal computed tomography (CT) scans. More significantly, patients with cancer infected with coronavirus were more likely to have an adverse event defined as admission to the intensive care unit requiring ventilation or death ( 39 vs. $8 \%, p=0.0003$ ). Cancer patients also had a shorter median time to severe events (13 vs. 43 days, $p<0.0001)$. Cancer, as a variable, had the highest odds ratio $[5,3]$ for having adverse events. Despite the limitation of a small sample size and the heterogeneity of cancer patients, these results are concerning. Cancers differ in their pathogenesis and the urgency of starting treatment is not the same for all cancers. We report our personal opinions regarding the management of patients with multiple myeloma (MM) during this challenging time. The reader is advised that these recommendations are based on experience and are not evidence based.

\section{What Is Special about MM Patients?}

The immune system in patients with MM is compromised by many different factors and patients with MM are at increased risk of infection [7]. The mean age at diagnosis for myeloma is approximately 65 and elderly patients are more likely to have comorbidities compromising the immune system. Lymphopenia at diagnosis is a well-known risk factor for developing infections [8-10]. This is a result of the myeloma clone suppressing normal B-cell development and function. A loss of functional immunoglobulins seen in most myeloma patients can also contribute to the increased risk of infection. Decreased $\mathrm{CD}+4 \mathrm{~T}$-cell count at diagnosis is also associated with increased infections $[11,12]$. Patients with MM receive treatment that causes more immunosuppression and increases the risk of infection [13]. Lymphopenia worsens after starting therapy for MM [10] and CD4 counts decrease with proteasome inhibitor (PI) therapy [14], and the risk of zoster reactivation is up to $13 \%$ without prophylaxis [15]. Immunomodulatory drugs (IMiDs) cause neutropenia, which increases the infection risk. Steroids are well known to increase the risk of infections (including pneumocystis and fungus) and the combination with IMiDs and PIs increases this further. The infection risk is highest during the first 3-4 months of therapy. Prophylactic levofloxacin for 12 weeks in newly diagnosed myeloma has been shown to reduce deaths significantly [16].
All of the previous factors make MM patients more susceptible to the serious adverse events that could arise with COVID-19 infection. COVID-19 can intensify neutropenia [17].

\section{What Is Our Approach to Smoldering MM?}

There is no disagreement regarding the management of standard-risk smoldering MM (SMM) patients. These patients should be monitored with no active intervention. For high-risk SMM based on the Mayo 2018 risk stratification [18], experts differ in their approach. Two phase III randomized trials evaluated the use of active therapy for SMM, one with lenalidomide and dexamethasone [19] and the other with lenalidomide alone [20]. There was improvement in progression-free survival (PFS) in 2 trials $[19,20]$ and overall survival (OS) in 1 trial [19]. However, both trials had several limitations. In both trials, the sample size was less than 100 patients in each arm. In the Spanish myeloma group trial [19], imaging was done using X-ray, which is less sensitive than current diagnostic modalities (including low-dose CT skeletal survey and PET scans), and the benefit of survival in some patients could have been due to treating active MM (normal radiographs but abnormal PET or MRI), rather than SMM. In the second trial, there is no OS benefit to date and only 30\% were high risk according to the Mayo 2018 risk stratification [20]. Also, 3.3\% of patients had bone marrow plasma cells $\geq 60$ and $8.2 \%$ had serum-free light chains $>100 \mathrm{mg} / \mathrm{dL}$ which are MM defining events by definition [20] and these patients may have been randomized to no therapy.

We currently believe that involving patients in a clinical trial is the best strategy for high-risk SMM patients. However, with the current COVID-19 pandemic, many trials are not accepting new patients and we would advise close monitoring and observation for these patients.

\section{What Is Our Approach to Newly Diagnosed Patients?}

\section{Risk Stratification}

MM is a heterogeneous malignancy and patients behave differently. Risk stratification is critical, especially during the current COVID-19 situation. In general, patients with trisomies, $\mathrm{t}(11 ; 14)$, or $\mathrm{t}(6 ; 14)$ are considered standard risk. High-risk genetic abnormalities include $\mathrm{t}(4 ; 14), \mathrm{t}(14 ; 16), \mathrm{t}(14 ; 20), 17 \mathrm{p}$ del, $1 \mathrm{q}+$ and $\mathrm{p} 53$ mutations. The Revised International Staging System (R-ISS) 
[21] defines patients with stage III as high risk. Patients with extramedullary disease are also considered high risk. We consider patients with plasma cell leukemia (PCL) as ultra-high risk.

\section{Screening for COVID-19 before Starting Therapy}

We recommend screening all patients with newly diagnosed MM for COVID-19 before starting therapy, as treatment for MM can aggravate the adverse events of an active COVID-19 infection. Screening should be done for outpatients as well as inpatients initiating therapy.

For patients testing positive for COVID-19 we recommend holding treatment until they have convalesced from acute illness. Metabolic and bone-related acute complications can be best managed with supportive care.

\section{Patient and Family Member Education}

Prevention is the best cure. We stress educating the patient about the impact of COVID-19 on their health, emphasizing the weak immune system in patients with MM. Social distancing, staying home (except for treatment), washing hands frequently with soap (for at least $20 \mathrm{~s}$ ), covering coughs and sneezes with tissue or using the elbow, and cleaning frequently touched surfaces are recommended [22].

Family members are critical for patient care and their mental health is important. Social support including tips for coping with stress should be provided to the patient and their family members. We find the following website (https://suicidepreventionlifeline.org/current-events/ supporting-your-emotional-well-being-during-the-covid-19-outbreak/) useful in providing recommendations regarding coping with the current pandemic [23].

\section{What Regimen?}

For Standard-Risk Patients

We believe that these patients could be managed using oral drugs to decrease the risk of weekly hospital visits and exposures. We recommend ixazomib, lenalidomide, and dexamethasone (IRd) given over a 28 -day cycle. Lenalidomide should be given at a dose of $25 \mathrm{mg}$ p.o. daily on days 1-21 and ixazomib at a dose of $4 \mathrm{mg}$ p.o. on days 1,8 , and 15 . We recommend decreasing the dose of dexamethasone to $20 \mathrm{mg}$ p.o. weekly to decrease the risk of infection. We understand that the IRd regimen has not been compared head to head with known standard therapy, but the response rate from the phase $1 / 2$ trial is comparable [24] and in our opinion using an oral regimen will help in decreasing the exposure to and infection with COVID-19.
Another possible combination is cyclophosphamide lenalidomide and dexamethasone (CRd) given over a 28day cycle [25] with cyclophosphamide $\left(300 \mathrm{mg} / \mathrm{m}^{2}\right.$ p.o. on days 1,8 , and 15$)$, lenalidomide ( $25 \mathrm{mg}$ p.o. on days 1-21), and dexamethasone (20 mg p.o. daily once weekly).

A third alternative is daratumumab lenalidomide and dexamethasone (DRd) given over a 28-day cycle [26]. Full doses of daratumumab and lenalidomide should be given. We would recommend that the first dose of dexamethasone is $40 \mathrm{mg}$ p.o. to decrease the risk of infusionrelated reaction to daratumumab. We would recommend giving dexamethasone orally, rather than intravenously, to decrease the total time in the chemotherapy unit for the patient. The dose of dexamethasone can be decreased during subsequent cycles, if no infusion reaction is documented. If no infusion reactions occur, the interval for daratumumab infusion can be decreased to $90 \mathrm{~min}$.

We understand that not all patients can access these medications. In that case, standard of care therapy using lenalidomide, bortezomib, and dexamethasone (RVd) should be considered. We would recommend RVd over 28 days with lenalidomide (25 mg p.o. on days 1-21), bortezomib $\left(1.5 \mathrm{mg} / \mathrm{m}^{2}\right.$ s.c. on days 1,8 , and15), and dexamethasone (20 mg p.o. daily once weekly). We want to stress the point that bortezomib should be given subcutaneously once a week, rather than intravenously or twice weekly. In some countries, cyclophosphamide, bortezomib, and dexamethasone (CyBorD) is the only approved combination for initial therapy. We again would recommend bortezomib to be given subcutaneously once a week and the dose of dexamethasone reduced to $20 \mathrm{mg}$.

\section{For High-Risk Patients}

Patients with high-risk features have a different biology and the management of these patients can be very challenging. We believe these patients have a greater risk of morbidity from myeloma than COVID-19 infection justifying weekly clinic visits for therapy, while in standard-risk patients an all oral regimen allows for therapy visits every 4 weeks. For high-risk patients, we would recommend carfilzomib, lenalidomide, and dexamethasone (KRd) given over 28 days for each cycle. We do not believe there is a high risk for tumor lysis syndrome with MM and we would recommend starting therapy for the first cycle with once-weekly dosing, rather than doing day 1 and day 2 infusions. If the patient has good bone marrow reserve, we would recommend carfilzomib at $70 \mathrm{mg} /$ 
$\mathrm{m}^{2}$ i.v. on days 1,8 , and 15 , lenalidomide $25 \mathrm{mg}$ p.o. on days 1-21, and dexamethasone $20 \mathrm{mg}$ p.o. once weekly. If there are concerns regarding cytopenias, we would recommend carfilzomib at a dose of 56 or $36 \mathrm{mg} / \mathrm{m}^{2}$ i.v. (as appropriate) on days 1,8 , and 15 . If KRd is not available, we would recommend $\mathrm{RVd}$, as above.

\section{Supportive Management}

Supportive measures are invaluable for patients with MM. IMiDs increase the risk of venous thromboembolism and prophylactic anticoagulation is needed. We recommend aspirin (325 mg p.o. daily) for patients with no other risk factors for venous thromboembolism. Other forms of anticoagulation are required in higher-risk patients. Prophylaxis against varicella-zoster virus (acyclovir $400 \mathrm{mg}$ p.o. b.i.d. daily) is required for patients on PIs. For Pneumocystis jirovecii prophylaxis, we would recommend using single-strength trimethoprim-sulfamethoxazole given daily or double strength given on Monday, Wednesday, and Friday. If the patient cannot safely receive sulfa, we would recommend using prophylaxis other than pentamidine, as its administration requires a clinic visit and the fact that it is an aerosolized therapy could be a concern with the current COVID-19 pandemic.

For bone disease prophylaxis, we believe that it can be delayed, especially in patients without active bone disease. If a patient has diffuse bone disease or has hypercalcemia, then we would recommend using zoledronic acid. For bone prophylaxis, this can be given every 3 months, rather than monthly. Patients should be advised to take calcium and vitamin D.

We do not recommend using growth factors. If a patient requires it, we would recommend using pegfilgrastim, if possible.

\section{Lab Work and Follow-Up}

We would recommend decreasing the frequency of blood work during each cycle as much as possible. If the patient has good bone marrow reserve, blood work can be done after finishing the cycle (before starting the next cycle), rather than checking counts routinely every week during the first cycle. We also recommend followup using telehealth technologies (telephone calls, video, etc.).

Drug companies have extended the supply of lenalidomide and pomalidomide to 8 weeks with drugs being shipped home, rather than requiring a visit to a specialized pharmacy. This will help reduce potential exposure to COVID-19.

Multiple Myeloma and COVID-19
The Hot Question: Should We Do Autologous Stem

Cell Transplant after Induction?

Autologous stem cell transplant (ASCT) clearly improves outcomes in newly diagnosed MM patients. However, during this challenging time, each patient should be evaluated individually. Multiple trials have evaluated upfront versus delayed ASCT and overall, upfront ASCT improves PFS, but not OS [27-31]. The two main concerns with the current COVID-19 pandemic is that patients need their stem cells collected and some patients require more than one apheresis. With conditioning comes the increased risk of infection related to the 7-9 days of severe neutropenia.

\section{Standard-Risk Patients}

For standard-risk patients who are responding well to therapy, we would recommend delaying ASCT until the current situation with COVID-19 improves. Continued induction therapy can safely be used as a bridge until the epidemic subsides [32].

\section{High-Risk Patients}

For high-risk patients, if they are responding (at least with partial response) ASCT can be delayed. However, patients who are not responding or progressing quickly, ASCT can be considered as salvage therapy. Testing for COVID-19 should be done before ASCT. Balancing the risks of COVID-19 infection and MM-related mortality is difficult. Consultation with a MM expert is recommended.

\section{Ultra-High-Risk Patients (PCL)}

These are the most challenging patients during the current pandemic. The median OS in these patients is less than a year with current treatment approaches [33]. We believe that induction chemotherapy followed by ASCT should be done for these patients, as the risk of progressive disease is high. Testing for COVID-19 should be done before ASCT.

\section{Any Concerns for Collecting Stem Cells Later?}

There are no major concerns for collection, as patients receiving IMiD-based therapy can successfully undergo stem cell collection after 6 cycles [34] using granulocyte colony-stimulating factor (G-CSF). For patients who fail to collect using G-CSF, plerixafor can be used.

Delaying stem cell collection is wise, especially in patients who had a recent COVID-19 infection. Hyperimmunity with cytokine storms and acute respiratory distress syndrome from the infection has been described [35, 36]. In theory G-CSF can reinitiate the immune stimulus leading to complications. 
How Many Cycles Should We Give/Any Dose

\section{Modifications?}

In general, we would recommend continuing with treatment until the current epidemic with COVID-19 improves and ASCT is safer.

Standard-Risk Patients

Patients who achieve sustained very good partial response or better ( $\geq$ VGPR) after 3-4 cycles, the doses of dexamethasone should be decreased further to $12 \mathrm{mg}$ with monitoring of response. If the concerns regarding COVID-19 continue, and the patient is on bortezomib, the frequency of administration can be decreased to every other week after $6-8$ cycles (if response is $\geq V G P R$ and is sustained). If the patient is on daratumumabbased therapy (DRd) and has achieved $\geq$ VGPR after the second cycle, we would recommend continuing the cycle every 28 days, but change the daratumumab to every 4 weeks rather than every 2 weeks. After 10-12 cycles and if the goals of therapy are achieved, switching to maintenance therapy with lenalidomide can be considered.

High-Risk Patients

Overall, we believe that these patients should continue on their triplet therapy for 10-12 months. The dose of dexamethasone can be decreased after 4-6 cycles if sustained deep responses $(\geq \mathrm{VGPR})$ are achieved. The frequency of PIs can be reduced to every 2 weeks, if the response is sustained. After 10-12 cycles, we would recommend PI-based maintenance with bortezomib subcutaneously every 2 weeks or weekly ixazomib. Those patients should undergo ASCT as soon as the pandemic resolves.

\section{Special Population: Frail/Non-Transplant-Eligible \\ Patients}

If DRd is available, we would recommend it for these patients if weekly clinic visits are feasible and safe. If not, using a triplet, such as IRd (preferred), CRd, RVd, or CyBorD can also be considered, depending on availability. These patients frequently require dose adjustments and close monitoring is recommended.

\section{What Is Our Approach to Progressing/Relapsing Patients?}

Most patients with MM will relapse and the management of relapse should be individualized depending on the patient, as not all relapses biologically behave the same. A discussion regarding the management of relapsed MM patients is beyond the scope of this review. We recommend viewing the mSMART guidelines from our institution [37]. Key points are described below:

\section{Standard-Risk Patients}

For those patients who relapse late from diagnosis with no high-risk genetic features and who did not receive daratumumab as part of the induction, we would recommend daratumumab-based regimens. If the patient responds well and is stable, recommendations regarding dose adjustment can be followed as for newly diagnosed patients (above). We would not recommend proceeding directly with ASCT (if it was part of the treatment plan) until the current pandemic resolves. If daratumumab is not available, other triplet combinations should be used depending on availability, fitness of the patient, and type of maintenance therapy. If the patient is not responding well and is failing conventional therapy, ASCT can be considered as salvage. A reminder: these patients should be screened for COVID-19 before ASCT.

\section{High-Risk Patients}

These patients are the most difficult to manage with the current situation. The management will depend on the nature of relapse and how aggressive it is. Again, if patients did not receive daratumumab, daratumumabbased treatment should be used. If daratumumab is not available, other triplet combinations should be considered depending on availability, fitness of the patient, and type of maintenance therapy.

The decision regarding ASCT should be individualized. For patients with aggressive relapse (especially with suboptimal responses to salvage therapy), ASCT should be considered as salvage. These patients should be screened for COVID-19 before ASCT. For patients who relapse slowly and achieve deep responses ( $\geq$ VGPR) after therapy, ASCT should be delayed.

\section{Ultra-High-Risk Patients}

Patients with secondary PCL (in the setting of refractory/relapsed MM) have a grim prognosis [38]. These patients should receive myeloma-directed therapy followed by ASCT. Testing for COVID-19 should be done before ASCT. 


\section{What Is Our Approach to Stable Myeloma Patients on Maintenance?}

We believe that patients who are stable on maintenance with no major side effects should continue on their maintenance. If the patient is on dexamethasone during their maintenance, we recommend tapering it down with the goal of discontinuing it. These patients do not need to be seen in clinic for 3 months. Monitoring should occur in the closest laboratory and phone visits may be used for toxicity checks.

\section{How about Clinical Trials?}

In many centers clinical trials have been suspended for new patients because the requirements for testing lead to frequent protocol violations. Some clinical trials are modifying their protocols, allowing blood draws to be done locally, telemedicine evaluation, and oral drugs to be shipped home.

\section{Conclusions}

The management of patients with MM during the current pandemic of COVID-19 is challenging. We do not recommend any active therapy for SMM patients, standard or high risk. Patients with active MM should be risk stratified before treatment. Screening for COVID-19 should be done for all patients (in patients and outpatients) before starting therapy. For standard-risk patients options include: IRd (preferred), CRd, DRd, RVd, or CyBorD. For high-risk patients KRd (preferred) or RVd are options. We recommend decreasing the dose of dexamethasone to 20 mg p.o. once weekly and the PIs should be given once weekly with bortezomib being given subcutaneously. Supportive management with antibiotics and anticoagulants (as needed) is critical. We recommend delaying ASCT, un- less the patient has high-risk disease that is not responding well, or if the patient has PCL. Testing for COVID-19 should be done before any ASCT or new chemotherapy regimen. Doses and frequency of drug administration can be modified, especially in patients with $\geq$ VGPR that is sustained. After 10-12 cycles, maintenance can be started with lenalidomide for standard-risk patients and bortezomib or ixazomib for high-risk patients. For relapsed patients, daratumumab-based regimens should be considered. We would recommend ASCT for patients with aggressive relapse, as well for patients with secondary PCL. Patients on maintenance should continue their therapy. In many centers clinical trials have been suspended as a result of the current pandemic with COVID-19.

\section{Disclosure Statement}

The authors have no conflicts of interest to declare related to this paper.

A.S.A.: no conflicts of interest to declare. T.S.: advisory board: Akcea, Alnylam, and Astrazeneca. Research support: InCyte and Prothena. M.A.G.: personal fees: Ionis/Akcea, Alnylam, Prothena, Celgene, Janssen, Spectrum, Annexon, Appellis, Research to Practice, Amgen, Medscape, Physicians Education Resource, and Abbvie. Grants: Spectrum, Amyloidosis Foundation, and International Waldenstrom Foundation. Speaker fees: Teva, Johnson and Johnson, Medscape, and DAVA oncology. Advisory board: Pharmacyclics and Proclara. Royalties: Springer Publishing. Development of educational material: i3Health. Stock options: Aurora Bio.

\section{Funding Sources}

The authors have no funding sources to declare.

\section{Author Contributions}

A.S.A., T.S., and M.A.G. contributed to the conception of the manuscript. A.S.A. wrote the first draft. T.S. and M.A.G. carefully reviewed the first draft and modified it. A.S.A., T.S., and M.A.G. approved the final revision.

\section{References}

Multiple Myeloma and COVID-19
1 World Health Organization [Internet]. Coronavirus disease (COVID-2019) situation reports [cited 2020 Mar 30]. Available from: https://www.who.int/emergencies/diseases/ novel-coronavirus-2019/situation-reports.

2 Pellino G, Spinelli A. How COVID-19 Outbreak is impacting colorectal cancer patients in Italy: a long shadow beyond infection. Dis Colon Rectum. 2020 Mar;1. Epub ahead of print.
3 Luo Y, Zhong M. [Standardized diagnosis and treatment of colorectal cancer during the outbreak of corona virus disease 2019 in Renji hospital]. Zhonghua Wei Chang Wai Ke Za Zhi. 2020 Mar;23(3):211-6. Chinese.

4 Ueda M, Martins R, Hendrie PC, McDonnell T, Crews JR, Wong TL, et al. Managing cancer care during the COVID-19 pandemic: agility and collaboration toward a common goal. J Natl Compr Canc Netw. 2020 Mar;18(4):1-4. 
5 Zhao Z, Bai H, Duan JC, Wang J. [Individualized treatment recommendations for lung cancer patients at different stages of treatment during the outbreak of 2019 novel coronavirus disease epidemic]. Zhonghua Zhong Liu Za Zhi. 2020 Mar;42(0):E007. Chinese.

6 Liang W, Guan W, Chen R, Wang W, Li J, Xu $\mathrm{K}$, et al. Cancer patients in SARS-CoV-2 infection: a nationwide analysis in China. Lancet Oncol. 2020 Mar;21(3):335-7.

7 Blimark C, Holmberg E, Mellqvist UH, Landgren $\mathrm{O}$, Björkholm $\mathrm{M}$, Hultcrantz $\mathrm{M}$, et al. Multiple myeloma and infections: a population-based study on 9253 multiple myeloma patients. Haematologica. 2015 Jan;100(1): $107-13$.

8 Shimizu Y, Inoue H, Hishinuma S, Shoji M. Nadir lymphocytopenia as a risk factor of bloodstream infection during molecular targeting pharmacotherapy in multiple myeloma. Int J Clin Pharmacol Ther. 2019 Nov; 57(11):542-51.

9 Hyun SY, Han SH, Kim SJ, Jang JE, Kim Y, $\mathrm{Cho} \mathrm{H}$, et al. Pretreatment lymphopenia, poor performance status, and early courses of therapy are risk factors for severe bacterial infection in patients with multiple myeloma during treatment with bortezomib-based regimens. J Korean Med Sci. 2016 Apr;31(4): 510-8.

10 Jung SH, Bae SY, Ahn JS, Kang SJ, Yang DH, Kim YK, et al. Lymphocytopenia is associated with an increased risk of severe infections in patients with multiple myeloma treated with bortezomib-based regimens. Int J Hematol. 2013 Mar;97(3):382-7.

11 Schütt P, Brandhorst D, Stellberg W, Poser M, Ebeling P, Müller S, et al. Immune parameters in multiple myeloma patients: influence of treatment and correlation with opportunistic infections. Leuk Lymphoma. 2006 Aug;47(8): 1570-82.

12 Lee SE, Lim JY, Ryu DB, Kim TW, Park SS, Jeon YW, etal.LowfrequencyofCD3+CD4+CD161+ $\mathrm{T}$ cells correlates with the occurrence of infections in refractory/relapsed multiple myeloma patients receiving lenalidomide plus low-dose dexamethasone treatment. Ann Hematol. 2018 Nov;97(11):2163-71.

13 Teh BW, Harrison SJ, Worth LJ, Thursky KA, Slavin MA. Infection risk with immunomodulatory and proteasome inhibitor-based therapies across treatment phases for multiple myeloma: a systematic review and meta-analysis. Eur J Cancer. 2016 Nov;67:21-37.

14 Heider U, Rademacher J, Kaiser M, Kleeberg L, von Metzler I, Sezer O. Decrease in CD4+ $\mathrm{T}$-cell counts in patients with multiple myeloma treated with bortezomib. Clin Lymphoma Myeloma Leuk. 2010 Apr;10(2):134-7.

15 Chanan-Khan A, Sonneveld P, Schuster MW, Stadtmauer EA, Facon T, Harousseau JL, et al. Analysis of herpes zoster events among bortezomib-treated patients in the phase III APEX study. J Clin Oncol. 2008 Oct;26(29):4784-90.

16 Drayson MT, Bowcock S, Planche T, Iqbal G, Pratt G, Yong K, et al.; TEAMM Trial Man- agement Group and Trial Investigators. Levofloxacin prophylaxis in patients with newly diagnosed myeloma (TEAMM): a multicentre, double-blind, placebo-controlled, randomised, phase 3 trial. Lancet Oncol. 2019 Dec;20(12):1760-72.

17 Huang C, Wang Y, Li X, Ren L, Zhao J, Hu Y, et al. Clinical features of patients infected with 2019 novel coronavirus in Wuhan, China. Lancet. 2020 Feb;395(10223):497-506.

18 Lakshman A, Rajkumar SV, Buadi FK, Binder M, Gertz MA, Lacy MQ, et al. Risk stratification of smoldering multiple myeloma incorporating revised IMWG diagnostic criteria. Blood Cancer J. 2018 Jun;8(6):59.

19 Mateos MV, Hernández MT, Giraldo P, de la Rubia J, de Arriba F, López Corral L, et al. Lenalidomide plus dexamethasone for high-risk smoldering multiple myeloma. N Engl J Med. 2013 Aug;369(5):438-47.

20 Lonial S, Jacobus S, Fonseca R, Weiss M, Kumar S, Orlowski RZ, et al. Randomized trial of lenalidomide versus observation in smoldering multiple myeloma. J Clin Oncol. 2020 Apr;38(11):1126-37.

21 Palumbo A, Avet-Loiseau H, Oliva S, Lokhorst HM, Goldschmidt H, Rosinol L, et al. Revised international staging system for multiple myeloma: a report from International Myeloma Working Group. J Clin Oncol. 2015 Sep;33(26):2863-9.

22 CDC [Internet]. Get your home ready: detailed planning guidance [cited $2020 \mathrm{Mar} 30$ ]. Available from: https://www.cdc.gov/coronavirus/ 2019-ncov/daily-life-coping/get-your-household-ready-for-COVID-19.html?CDC_AA refVal=https $\% 3 \mathrm{~A} \% 2 \mathrm{~F} \% 2 \mathrm{Fwww} . \mathrm{cdc}$. gov\% 2 Fcoronavirus\%2F 2019 -ncov\%2Fprepare\%2Fget-your-household-ready-forCOVID-19.html.

23 Suicide Prevention Lifeline [Internet]. Emotional wellbeing during the COVID-19 outbreak[cited 2020 Mar 30]. Available from: https://suicidepreventionlifeline.org/current-events/supporting-your-emotionalwell-being-during-the-covid-19-outbreak/.

24 Kumar SK, Berdeja JG, Niesvizky R, Lonial S, Laubach JP, Hamadani M, et al. Ixazomib, lenalidomide, and dexamethasone in patients with newly diagnosed multiple myeloma: long-term follow-up including ixazomib maintenance. Leukemia. 2019 Jul;33(7): 1736-46.

25 Kumar SK, Lacy MQ, Hayman SR, Stewart K, Buadi FK, Allred J, et al. Lenalidomide, cyclophosphamide and dexamethasone (CRd) for newly diagnosed multiple myeloma: results from a phase 2 trial. Am J Hematol. 2011 Aug; 86(8):640-5.

26 Facon T, Kumar S, Plesner T, Orlowski RZ, Moreau P, Bahlis N, et al.; MAIA Trial Investigators. Daratumumab plus lenalidomide and dexamethasone for untreated myeloma. N Engl J Med. 2019 May;380(22):2104-15.

27 Fermand JP, Ravaud P, Chevret S, Divine M, Leblond V, Belanger C, et al. High-dose therapy and autologous peripheral blood stem cell transplantation in multiple myeloma: upfront or rescue treatment? Results of a multicenter sequential randomized clinical trial. Blood. 1998 Nov;92(9):3131-6.

28 Attal M, Lauwers-Cances V, Hulin C, Leleu X, Caillot D, Escoffre M, et al.; IFM 2009 Study. Lenalidomide, Bortezomib, and Dexamethasone with Transplantation for Myeloma. N Engl J Med. 2017 Apr;376(14):1311-20.

29 Kumar SK, Lacy MQ, Dispenzieri A, Buadi FK, Hayman SR, Dingli D, et al. Early versus delayed autologous transplantation after immunomodulatory agents-based induction therapy in patients with newly diagnosed multiple myeloma. Cancer. 2012 Mar;118(6):1585-92.

30 Remenyi P, Varga G, Mikala G, Reti M, Gopcsa L, Batai A, et al. Early versus delayed autologous stem cell transplantation and interferon maintenance in multiple myeloma: single-center experience of 18 Years. Transplant Proc. 2016 Jan-Feb;48(1):177-84.

31 Dunavin NC, Wei L, Elder P, Phillips GS, Benson DM Jr, Hofmeister CC, et al. Early versus delayed autologous stem cell transplant in patients receiving novel therapies for multiple myeloma. Leuk Lymphoma. 2013 Aug;54(8):1658-64.

32 Chakraborty R, Muchtar E, Kumar SK, Buadi FK, Dingli D, Dispenzieri A, et al. Impact of duration of induction therapy on survival in newly diagnosed multiple myeloma patients undergoing upfront autologous stem cell transplantation. Br J Haematol. $2018 \mathrm{Jul}$; 182(1):71-7.

33 Ravi P, Kumar SK, Roeker L, Gonsalves W, Buadi F, Lacy MQ, et al. Revised diagnostic criteria for plasma cell leukemia: results of a Mayo Clinic study with comparison of outcomes to multiple myeloma. Blood Cancer J. 2018 Nov;8(12): 116.

34 Kumar S, Dispenzieri A, Lacy MQ, Hayman SR, Buadi FK, Gastineau DA, et al. Impact of lenalidomide therapy on stem cell mobilization and engraftment post-peripheral blood stem cell transplantation in patients with newly diagnosed myeloma. Leukemia. 2007 Sep;21(9):2035-42.

35 Chen C, Zhang XR, Ju ZY, He WF. [Advances in the research of cytokine storm mechanism induced by Corona Virus Disease 2019 and the corresponding immunotherapies]. Zhonghua Shao Shang Za Zhi. 2020 Mar;36(0): E005. Chinese.

36 Xu Z, Shi L, Wang Y, Zhang J, Huang L, Zhang $\mathrm{C}$, et al. Pathological findings of COVID-19 associated with acute respiratory distress syndrome. Lancet Respir Med. 2020 Apr; $8(4): 420-2$.

37 mSMART [Internet]. Treatment guidelines: multiple myeloma[cited 2020 Mar 30]. Available from: https://www.msmart.org/mmtreatment-guidelines.

38 Tiedemann RE, Gonzalez-Paz N, Kyle RA, Santana-Davila R, Price-Troska T, Van Wier SA, et al. Genetic aberrations and survival in plasma cell leukemia. Leukemia. 2008 May; 22(5):1044-52. 